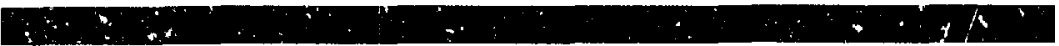

\title{
DISCUSSIONS OF BULK ATOMIC OR MOLECULAR SEPARATIONS BY RESONANT RADIATION PRESSURE
}

Edgar A. Rinehart

Jeffery if. Richardson

Lawrence W. Hrubesh

May 29, 1975

Prepared for U.S. Energy Fesearch \& Development

Administration under contract No. W-7405-Eng-48

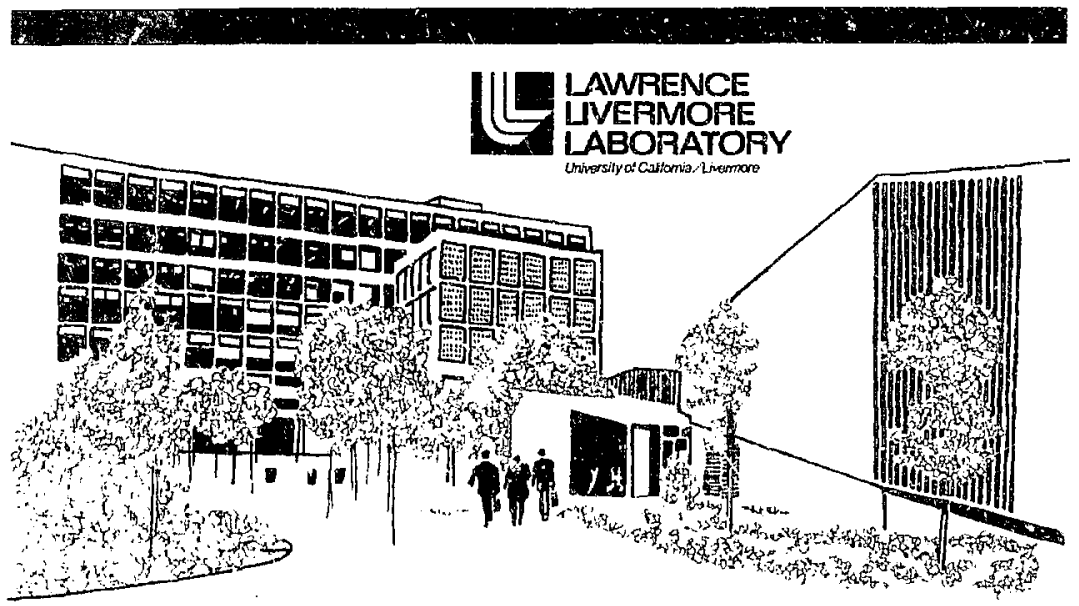

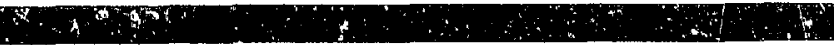


NOTICE

"This repar! was prepared as an account of work sponsored by the Unjted Stutes Government. Neither the United States nor ,he United Stutes Energy Resench \& Development Administrution, nor any of their employess, nor any of their contractors subcontractors, or their empluyeus, makes uny warranty, express or iniplied, or assumes any Iegit linbility of responsibility fot the aeturacy. completeness or usefulness of any information. apoatatus, product or process disciused, or repfosents that its use would bot infringe privately-uvned rights."

Printed in the United States of America Available from

National Technical Information Service U.S. Department of Commerce 5285 Port Royal Road Springfield, Virginia 22151

Price: Printed Copy $\$ *$ : Microfiche $\$ 2,25$

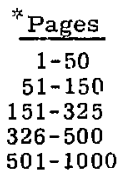

NTIS

Selling Price

$\$ 4.00$

$\$ 5.45$

$\$ 7.60$

$\$ 10.60$

$\$ 13.60$ 


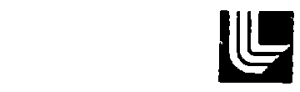

\title{
LAWRENCE LNERMORE LABORATOAY
}

Uniwsity of Culomia/Livermora, Cavtomin/ 94550

\section{UCRL-51833 \\ DISCUSSIONS OF BULK ATOMIC OR MOLECULAR SEPARATIONS BY RESONANT RADIATION PRESSURE}

\author{
Edgar A, Rinehart* \\ Jeffery H. Hichardson \\ Lawrence $W$. Hrubesh
}

MS. date: May 29, 1975

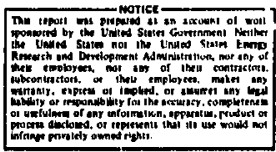

"Permanent address, University of Wyoming, Laramie, Wyoming.

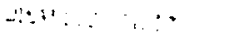




\section{Contents}

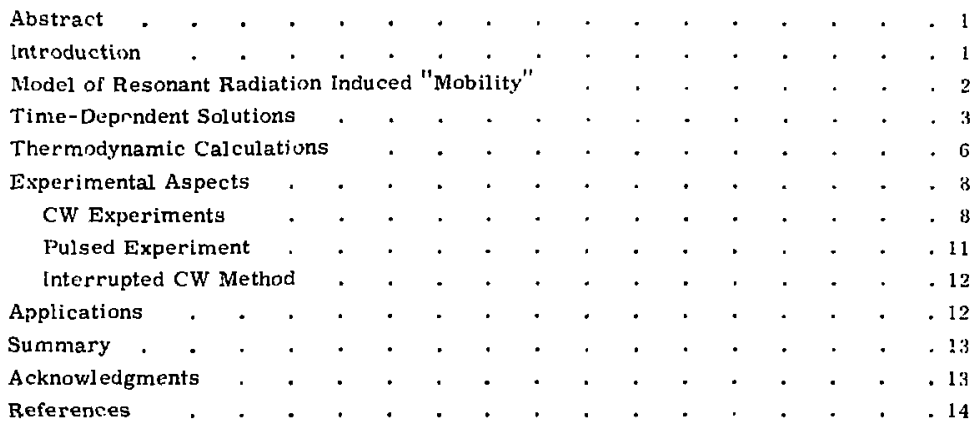




\section{DISCUSSIONS OF BULK ATOMIC OR MOLECULAR SEPARATIONS BY RESONANT RADIATION PRESSURE}

\begin{abstract}
Resonant radiation pressure has been produced by the transfer of photon momentum to an atom or molecule durinr resonant absorption of photon energy. We believe that this pressure is large enough to induce atomic and molecular separations when a laser is used as a source, We developed a mode] to deicribe the motion of molecules (or atoms) with a diffusionlimiter mobility, under the influence of laser-inrluced resonant radiation pressure. The time dependency of the
\end{abstract}

Abstract process was examined for a cw laser in the bulk movement of a one-component gas. Thermodynamic calculations showed that the process should be efitcient and substantial. Prelininary experimental results were not conclusive. Two additional experimental techniques have been examined: the use of a pulsating laser source; and the use of periodic withdrawals and replenishings of the mixture within the system. Applications in the bulk separations of isotopic mixtures and optical isomers are discussed.

\section{Introduction}

Considerable attention is being given to methods of inducing physical separations of atoms or molecules which normally exist in mixed composites. Particular emphasis is placed or isotopic separation, although chemical separations of any kind find many applications in present technology. One interesting approach to achieve atoml or molecular soparations, especially because of the potential use of lasers, is by resonant radiation pressure. Resonant radiation pressure is produced by the transfer of photon momentum to an atom or molecule during resonant absorption of photon energy. Ashkin ${ }^{1}$ has calculated that this pressure can indeed be large enough to induce atomic deparation when a laser is used as a source, and proposed that it might be used to separate isotopes. The principle has in fact been used by several experimencors ${ }^{2-4}$ to deflect resonant atoms from a beam. Although these experiments suggest the feasibility of this technique for separation, they all have used beams which considerably limit the yield of separated material. We will consider in this paper the possibility of separation of much larger quantities by applying resonant radiation pressure in a bulk process.

We will de elop a model which describes the motion of molecules (a similar case exista for atoms ) under the influence of the resonant radiation pressure and $\mathrm{B}$ 
"mobility" which is diffusion limited. A similar description of radiation induced pressure on a bulk gas was given recently by Gelbewachs and Hartwick. 5 The time dependence of the process will be examined for the case where $\mathrm{cw}$ laser radiation supplies the radiation pressure. Thermodynamic calculations are made for a binary mixture to predict the equilibrium state of such a system. We describe a sct of experiments designed to produce and observe the actual separation of gases and isotopes by this method. We discuss the optimization of experimental conditions and parameters, and de.cribe in detail a novel application of a pulsed laser which should produce highly efficient laserinduced separation in the bulk.

\section{Model of Resonant Radiation Induced "Mobility"}

We consicier an ideal, equilibrated gas, confine $\downarrow$ to $a$ long cylindrical tube of narrow cross-section at a reduced pressure, with a concentration of $n_{0}$ molecules/cc.

Suppose we could apply a force (F) uniformly over the cross-sectional area of the tube in the logitudinal $(\hat{x})$ direction which would affect the motion of a certain number ( $n$ ) of the molecules in the tube. In the absence of a restoring force, we would expect a net molecular flux through an element of area directed along $\hat{x}$ given by

$$
j_{x}=n \bar{v}_{x}
$$

where $\bar{v}_{x}$ is the everage drift velocity of the $\mathrm{n}$ molecules in the $\hat{\mathrm{x}}$ direction.

Opposing this molecular fux would be a flux in the opposite direction operating under a diffusional force. This flux can be given by

$$
j_{-x}=-D \text { An } / \theta x
$$

where $D$ is the self-diffusion constant and $8 \mathrm{n} / \mathrm{ax}$ is the molecular gradient in the $\hat{x}$ directior.

After a suffictent time, a steady state would be reached at which

$$
j_{x}=j_{-x}
$$

or

$$
\text { -D } \partial \mathrm{n} / \partial \mathrm{x}=\mathrm{n} \bar{v}_{\mathrm{x}} \text {. }
$$

The steady-state solution of this equation is

$$
n(x)=n_{0} \exp \left(-\bar{v}_{x} x / D\right)
$$

which is similar to that written by Ashkin. ${ }^{1}$ It implies that if the force is sufficiently large to impart a large mean drift volocity to the molecules in the $\hat{x}$ direction, the distribution of molecules within the tube along the $\hat{x}$ direction becomes expunential. Thus, as long as the applied force is maintained, molecules can be "pushed" toward one end of the tube and held there.

The force referred to above can bc supplied by the time rate of momentum transferred from a photon beam to molecules absorbing the photons during the resonant absorption process. Because only certain molecules can absorb those photons, they alone receive the preferential force, thus providing a mechanism to "push" certain molecular species to one end of the tubc. This phenomeron is called resonant radiation pressure or photon pressure.

For a more detalled understanding of the force produced by i'esonant radiation, 
ronsider a molecular system whose energy

levels are defined by a two-manifold vibrational-rotational structure. The resonant radiation is going to change the lower state $\left(n_{1}\right)$ and excited state $\left(n_{2}\right)$ populations of some transition within the manifold. The system's response will be determined by a combination of rates of stimulated absorption, radiative momentum transfer, collisional deactivation, and the rolaxation of the excited state by spontancous emissions. The effective radiation pressure force per molecule produced during resonant absc. ption is given by the product of the momentum per photon and the number of quanta absorbed per molecule per second. Thus,

$$
F_{\text {eff }}=(h / \lambda)(\gamma / / \Delta n)
$$

where $h, \lambda$ is the momentum per photon, $i$ is the urisaturated coefficient of absorption of the gas made up of $n$ molecules, $c$, $\Delta n$ is the difference in populations of the Lwo states involved, and $I$ is the radiation intensity (cxpressed in units of quanta per. second per unit cross-section of area). The torm $\left(\gamma_{i} ; \Delta n\right)$ repressints an effective deactivation rate of the bipper state of the transition sis that we can define an effective lifetime ( $\tau_{\text {eff }}$ ) for the upper state af

$$
\tau_{\text {eff }}=\left\{\gamma|/ \Delta n\rangle^{-1}\right. \text {. }
$$

The effective force per molecule then becone:s

$$
v_{\text {eff }}=\langle h / \lambda) \tau_{\text {eff }}^{-1} .
$$

We may consider the molecules to have a "mobility" under the action of the effective force $F_{\text {eff }}$ givan by

$$
\mu=v_{x} / F_{\text {eff }}
$$

where $v_{x}$ is the drift velocity defined above. From kinetic theor: the "mobility" is also defined by the "Einstein relation,"

$$
\mu=\mathrm{D} / \mathrm{kT}
$$

where $\boldsymbol{D}$ is the dirsusion coufficient. Using the equipartition principle and the definition of $D$ from kinetic theory

$$
\mu=\tau_{k} \cdot \mathbf{M}
$$

where $\tau_{k}$ is the mean time between collisions which transfer kinetic energy from the excited molecules and $M$ is their molecular woight. Then, from Eqs. (8), (9) and (11),

$$
v_{x}=(h / M \lambda)\left(\tau_{k} / \tau_{e f f}\right)
$$

which defines the drift velocity imparted to the molecules in the $\hat{\mathbf{x}}$ direetion in terms of the collisional and radiative lifetimes of the excited molecular state. F. Im En. (5), maximization of the drift veiocity $v_{x}$ optimizes the attainable spatial separation within the tube. Thus, to affect large separations, Toff shouid be made as small as possible (i.e., the deactivation of the upper state slrould be enlaticed).

\section{Time-Dependent Solutions}

An istimate of the time-dependence for molecular separation may be made by studying the vase for a one-component gas subject to resonant radiation pressure and self-diffusion. Consider a cyindrical tube of length $I$ and cross-section $A$ to 
be filled with a given gas at initial concentration $\left(C_{0}\right)$ and temperature $(T)$. Assume that the laser bcam fills the tube and that the photon fux is sufficiently high so that the rate-limiting step for absorption is the relaxation rate from the excited state to the ground state. Then, as previously defined, a drift velocity $v_{x}$ can be associated with the absorbing gas:

$$
r_{x}=(h / N D)\left(\tau_{k} i \tau_{e f f}\right) \text {. }
$$

Inder isothermal conditions and neglecting radial diffusion and wall offects (i,e., the mean free path is much less than the tube dianeter), the concentration $C(x, t)$ is given by the solution to

$\frac{\partial C(x, t)}{\partial t}=D \frac{\partial^{2} C(x, t)}{\partial x^{2}}-v_{x} \frac{\partial C(s, t)}{\partial x}$.

Two adelitional assumptions are made:

(1) an ideal gas is assumed, and (2) D, the diffusion coefficicnt, is assumed to be constant. Note that Fq. (14) is analogous to that if forced diffusion for a single gas mixture."

Fquation (1-t) can be solved by separa$t i m$ of variables subject to the following boundary conditions:

1) as $t$ approaches infintty, $C(x, t)$ approaches the steady-state solution, l.e.,

$$
\frac{\partial^{2} C^{\prime}(x, t)}{\partial s^{2}}-\frac{v x}{\partial} \frac{\partial C(x, t)}{\partial x}=0
$$

2) for the steady-gtate solution, $(\mathrm{C})=\mathrm{C}_{\mathrm{o}}$

3) at $t=0, C(x, t)=C_{0}$ for $0 \leq x \leq L$.

In addition, in order to obtain a closed analytleal solution, one of the following two assumptions inust be made:
4) $C=0$ at $x-0,1$, for $t=0 \mathrm{cos}$

a) $\frac{\partial C}{\partial \bar{x}}=0$ at $x=0$, I. for $t=0$.

Assumption (4) loads to the following expression for the concentration

$$
\begin{aligned}
& C(x, t)=\exp (2 \beta x) \\
& +\sum_{n=0}^{\infty} A_{n} \sin \frac{n \pi x}{L} \exp (i 3 x) \exp \left(-\lambda_{n}^{2}() t\right)
\end{aligned}
$$

where

$$
\begin{aligned}
& \mathrm{E}=\frac{2 ; 3 \mathrm{~L} \mathrm{C}_{0}}{\exp (2 \beta L L)-1} \\
& \beta={ } / 2 \mathrm{x})
\end{aligned}
$$

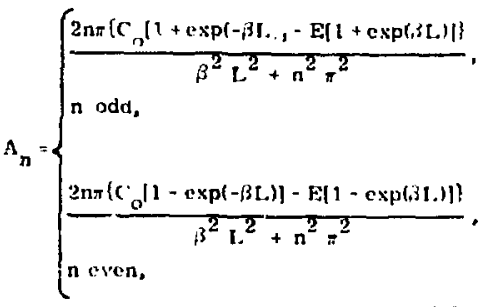

$$
\begin{aligned}
& \lambda_{n}^{2}=(n \pi / 1)^{2}+\beta^{2}
\end{aligned}
$$

This solution is more properly limited to the region $0<x<1$. As it approaries infinity, C approaches the steady-state solution Eexp(2/3x). This exponential variation of the concentration with distance was shown in 1E. (5) and previously obtained. 5,6 (Note that $\beta$ has the dimension (length $)^{-1}$.) The concentration approaches the steady-state solution with a time constant proportional to $\left(\lambda_{n}^{2} D\right)^{-1}$.

The alternate boundary condition, assumption (5), leads to the following expression for $C$ : 
$C(x, 1)=\operatorname{liexp}(2 \beta x)$

$+\sum_{n=0}^{\infty}\left[\left(A_{n}+F_{n}\right) \cos \frac{n \pi x}{L}\right.$

$\left.+i\left(A_{n}-B_{n}\right) \cdot \sin \frac{n \pi x}{T}\right] \exp (B x) \exp \left(-\lambda{ }_{n}^{2} D t\right)$

(21)

where $\mathrm{I}$ : and $\lambda_{n}^{2}$ arc defined as before. The steady-state solution falls out as a special case of the time dependent solution. Note that brth poundary conditions lead to identical expressions for the time dependence. This would be expected from comparison with other diffusion problems. ${ }^{6}$

The concentration must be real; a special case would be to require $A_{n}=B_{n}$. leading to the following expression for $c(x, t)$ :

$C(x, t) \quad \operatorname{Eexp}(2 \beta x)$

$$
+\sum_{n=0}^{\infty} A_{n} \cos \frac{\pi x}{\pi} \cos (\beta x) \exp \left(-\lambda_{n}^{2} D t\right)
$$

whore

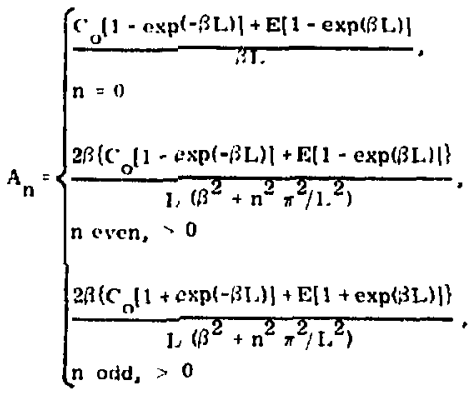

The most general solution is, of course, when $A_{n}=13_{n}^{*}$ (i.e., complex conjugates). However, closed analytical expressions for the coefficients cannot be obtalned in that case.

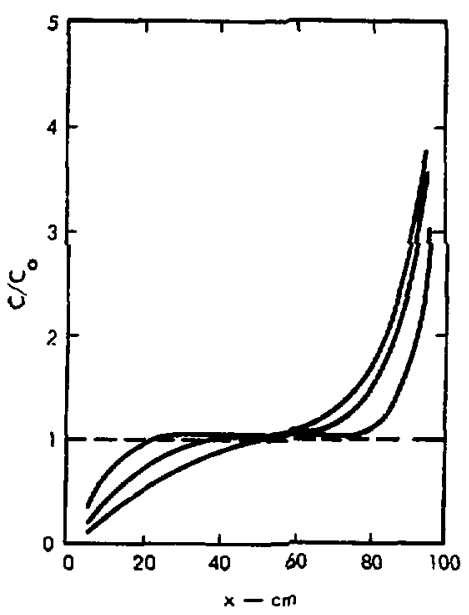

Fig. 1. Graphed solution to Eq. (16) (sin solution).

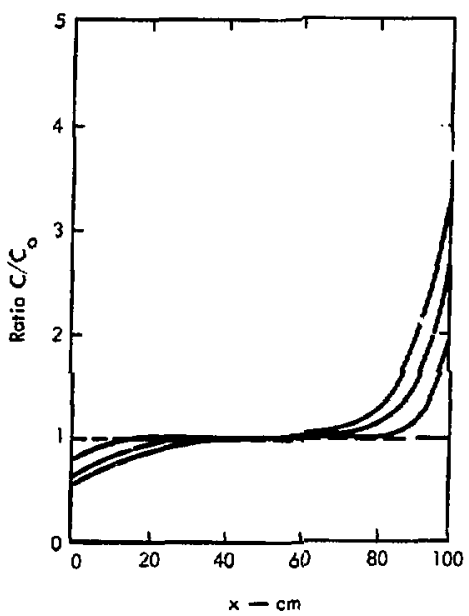

Fig. 2. Graphed solution to Eq. (21a) (cos solution). 
Figures 1 and 2 illustrate the two different expressions for $C(x, t)$ (Eqs. (16) and (21a) respectively]. For illustrative purpose the following values were used for the constants: $D=0.2 \mathrm{~cm}^{2} / \mathrm{sec}$, $v_{\mathrm{X}}=0.01 \mathrm{~cm} / \mathrm{sec}, \mathrm{L}=100 \mathrm{~cm}$, and $C_{0}=1$. The first 5000 terms were evaluated in the infinite sum! Eq. (2la) converges faster because of its $1 / n^{2}$ dependence. Although the expressions for the time dependence are identical, for this particular example Eq. (16) approaches the steady-state solution faster because $L$ is small and ali the terms for $\mathbf{n}=\mathbf{0}$ vanish.

Figure 3 represents the separation ratio $1 \mathrm{~cm}$ from the end of a $1 \mathrm{~m}$ tube. It is seen that the rate of separation decreases with time. For $0<\mathrm{t}<\infty$ the time varying

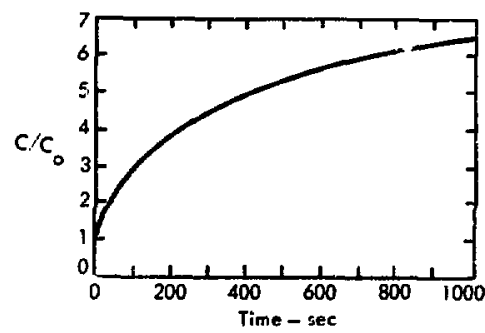

Fig. 3. Separation ratio at end of tube.

part of the solution does not integrate to zero. The data in Fig. 3 have been arcordingly renormalized; for a $1 \mathrm{~m}$ tube the maximum total error is ao sut $25^{\circ}$ at $\mathbf{5 0 0 0}$ seronds.

\section{Thermodynamic Calculations}

The degr ee of separation for a binary mixture can be estimated by considering the thermodynamics of mixing in a constant volume system. It will be apparent that the situation is simillar to that of osmosis, Assume A molecules are being separated by a laser from B molecules in a tube of volume $2 \mathrm{~V}$. As a: initial simplification, assume that the iaser changes the distribution funstion of $A$ from a constiut to a step function. Assume also that the experiment is performed under isothermal conditions, Initially both $\mathrm{A}$ and $\mathrm{B}$ molecules have a uniform distribution (Fig. 4a). After the laser is turned on, all the $A$ molecules are in the right hand side (Fig. 4b). The free energy $G(T)$, is a atate function, and hence the path chosen to reach equilibrium wlll have no effect on the equiltbrium distribution. a)

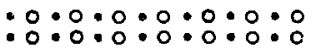

initial uniform distribution of $A+B$

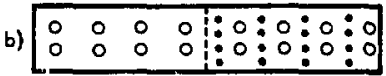

Distribution after laser activation

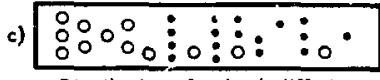

Distribution after back diffusion

- A molecules

- B molecules

Fig. 4. Schematic representation of binary mixture sepa:ation. (a) Inttial uniform distribution of $A$ and $B$, (b) Distribution after laser activation. (c) Distribution after back diffusion. 
The question we wish to answer is which way and t" what extent will the $F$ molecules diffuse?

The charge in free energy $(\Delta G)$ is a function of the chemical potential, $\omega$, temperature, and pressure:

$$
\mathrm{dG}=-S \mathrm{dt} \cdot V \mathrm{~d} \mathrm{H}^{3}+\Sigma_{\mathrm{j}} \mathrm{rin}_{\mathrm{j}} \cdot
$$

We have assumed a distribution function for A whith is detrrmined by the work being done in $A$ by the laser. At cquilibrium therefure, $d G=0$ and is a function of $\mu_{K}$, $\mu_{H}^{R}$, and $\mathrm{V} d \mathrm{l}$.

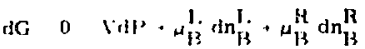

$\mu_{13}^{1}: \mu_{B}^{O} \cdot \operatorname{RT} \ln P_{13}^{l}$

$\mu_{B}^{R}=\mu_{B}^{O}+R T$ in $P_{B}^{R}$

$u_{\beta}^{\prime \prime}+R T \ln P_{T}^{R}+R T \ln X_{B}$

where $x_{B 3}$ is the mole fraction of $B$, $\mathrm{dn}_{B} \mathrm{~J}_{\mathrm{j}}$ the increase in moles of $B$ on the

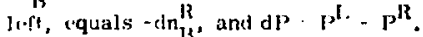
Niste that $\mu_{\mathbb{B}}^{\mathrm{l}} \quad \mu_{\mathbb{R}}^{\mathrm{R}}$, hence there will be no tendery for $B$ to separate duc to a differenew in thentical potential. This differs from osmosis, which normally applies to (ondensed phases and constant pressure. Assuming ideal gas behavior and substituting, Fq. (23) simpli "ies to the following:

$2 n_{A}-2 d n_{B}^{I}=\ln \left(\frac{n_{B, i}+d n_{B}^{J}}{n_{B, i}-d n_{B}^{I}}\right) d n_{B}^{I}$

where $n_{B, i}$ is the initial concentration of B. For a 1-liter tube at a total pressure of 2 Torr, equimolar mixture of $\Lambda$ and $B$, $d n_{B}^{L} / n_{B, j}=+70 \%$. Hence $B$ will back diffuse, further enhancing the degree of separation in an attrmpt to equalize the pressure (Fig. $4 \mathrm{c}$ ).

Equation (24) can be generalized to our specific case, assuming the steady-state we-compronent solution for $C_{A}(x)=\operatorname{Eexp}(23 x)$.

$$
\begin{aligned}
& 2\left[\operatorname{Eexp}(2 \beta x)-2 d c_{1}^{1}(x)\right] \\
& =\ln \left\{\left[\frac{C_{B, i} \cdot C_{B} C^{I}(x)}{C_{B, i}-C_{B H}(x)}\right]\right\} d C_{B}^{I}(x)
\end{aligned}
$$

This expression can be solved numerically to yirle $C_{B}(x)$ (Fig. Sl.

A calculation for the energy needed to overerme the entropy of mixing for a fully separated binary mixture shows that ideally (no energy lost in the process) $172(\mathrm{~J}$ mole are required to fully separate a hinary mixture.

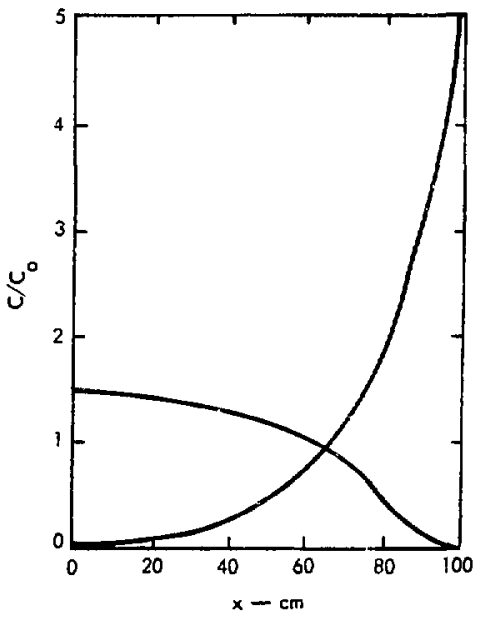

Fig. 5. Binary equilibrium distribution. 


\section{Experimental Aspects}

The ideal $\mathrm{cw}$ experiment to test for separation of molecular species would utilize a very short wavelength to maximize the drift velocity, sufficient power to saturate the transition to the point of maximum power absorption, a buffer gas which would rapidly remove molecules from the upper state, and, for initial tests, a gas with a very small diffusion coefficient. These conditions obviously cannot all be optimized. Ashkin ${ }^{1}$ has selected, for purposes of illustration, sodium vapor buffered by helium. Others ${ }^{5}$ have calculated the separation efficiencies for several spectral regions and a few small molecules. Separation efficiencies should not be based on input laser power, but on total expended energy. From that standpoint, the $\mathrm{CO}_{2}$ laser would be a reasonable choice for large numbers of cheap photons because it is the most efficient laser available today. Since many molecules have an absorption near one of the $\mathrm{CO}_{2}$ wavelengths, the concept would appear worthy of test.

Two slightly different approaches have bcen used in preliminary investigations. The results of the time-dependence solution in the proposed model, as shown in Fig. 3, suggest an approach which we will call the interrupted cw method. Recent results ${ }^{7}$ of studies of transient absorption of radiation suggest a pulsed technique, which will be discussed separately.

\section{CW EXPERIMENTS}

Very preliminary experiments at the University of Wyoming and more recent studies $\mathrm{a}_{i}$ : : L have utilized a $\mathrm{CO}_{2}$ laser cperating in the $\mathrm{cw}$ mode at a power output of 10 to $20 \mathrm{~W}$. The output beam irradiates a long tube containing a gaseous mixture at pressure of 0.1 to 100 Torr. In the earlier experiments, no wavelength selection was used, and the glow discharge (of $1 \mathrm{~m}$ length) was produced by a neon sign transformer. In these experiments, the copper absorption cell was $3,81 \mathrm{~m}$ long and $0.95 \mathrm{~cm} \mathrm{i.d.} \mathrm{The} \mathrm{laser} \mathrm{produced} \mathrm{a}$ beam of approximately $1.5 \mathrm{~cm}$ diam and filled the cross-section of the tube. In the later series of tests, a stabilized, wavelength selected laser was used, also of $1 \mathrm{~m}$ active length. A filtered dc supply was used to excite the discharge. The stainless steel cell length was $3.65 \mathrm{~m}$ and $0.95 \mathrm{~cm}$ in diameter. The beam diameter was approximately $0.5 \mathrm{~cm}$.

The procedure in both sets of experiments was similar: a small amount of an absorber was admitted, and a larger amount of some buffer was added. After allowing lime for the mixture to reach equilibrium, the laser was turned on for a time which varied from $15 \mathrm{~min}$ to $4 \mathrm{hr}$. Sample bottles at each end of the tube were then valved off and the samples analyzed by either microwave spectroscopy or mass spectroscopy.

The test gases used were ${ }^{14} \mathrm{NH}_{3}, 15_{\mathrm{NH}_{3}}$, and $\mathrm{CH}_{2} \mathrm{Cl}-\mathrm{CH}_{2} \mathrm{Cl}$ with $\mathrm{N}_{2}$, air, and $\mathrm{SO}_{2}$ used as buffer gases. In the experiments discussed here, one or more reference samples were taken before irradiation to serve as a standard for analysis. 'These experiments have served primarily as indicators of experimental problems.

In an carlier experiment a binary mixture consisting of equal amnunts of $\mathrm{NH}_{3}$ and $\mathrm{SO}_{2}$ was admitted to the absorption 
Table 1. Microwave spectrometric analysis of test samples.

\begin{tabular}{|c|c|c|c|c|}
\hline & $\begin{array}{c}\mathrm{NH}_{3} \\
\text { Signal } \\
\text { Pressure } \\
\frac{\text { mv }}{\text { m Torr }}\end{array}$ & $\begin{array}{c}\text { Partial pressure } \\
\text { ratios } \\
\mathrm{NH}_{3} \text { in sample }\end{array}$ & $\begin{array}{c}\mathrm{SO}_{2} \\
\text { Signal } \\
\text { Pressure } \\
\frac{m y}{\text { m Torr }}\end{array}$ & $\begin{array}{c}\mathrm{SO}_{2} \\
\underset{\text { Partial pressure }}{\text { ratio }}\end{array}$ \\
\hline Refercnce & 0.639 & 1 & 3.14 & 1 \\
\hline Near sample & 0.117 & 0.184 & 3.564 & 1.044 \\
\hline Far sample & 0.428 & 0.671 & 3.557 & 1.042 \\
\hline
\end{tabular}

cell at a total pressure of 0.4 Torr. A reference sample $(125 \mathrm{cc})$ was taken, and laser irradiation oroceeded for $30 \mathrm{~min}$ at approximately $12 \mathrm{~W}$ with no wavelength selection.

A summary of observed results, using microwave spectrometric alalysis, is shown in Table 1. The loss of $\mathrm{NH}_{3}$, apparently to the walls, indicates one serious experimental pitfall. In another earlier experiment, the refer ence sample was not used, but the experiment was repeated, without irradiation, as a control. In this ease 1-2 dichloroethane was used with air as a buffer. A summary of the microwave -pectrometric analysis is shown in Table 2. The signal/pressure ratio is proportional to the concentration in the spectrometer. The experimental error in reading the pressure gauge used in this experinient is

Table 2. Reference famples taken without irradiation in binary separation experiment of $15 \mathrm{~m}$ Tarr $\mathrm{C}_{2} \mathrm{Cl}_{2} \mathrm{H}_{4}$ and 3 Torr air.

\begin{tabular}{lcc}
\hline & $\begin{array}{c}20 \mathrm{~min} \\
\text { irradiation } \\
(\mu \mathrm{V} / \mathrm{m} \text { Tor })\end{array}$ & $\begin{array}{c}\text { wait with no } \\
\text { irradiation } \\
(\mu \mathrm{V} / \mathrm{m} \text { Torr })\end{array}$ \\
\hline Near sample & 3.11 & 8 \\
Far sample & 48 & 3.4 \\
\hline
\end{tabular}

sufficient to account for the inequality of the second set of ratios.

The second series of experiments, at LLL, followed a similar procedure with important differences. The reference samples were taken for analysis from both the near and far ends before irradiation commenced. This was to ensure that thorough mixing of the gases had occurred. A second difference was that the sample bottles were of much larger volume than the active region.

The results of the second series of experiments were inconclusive due to difficulties in achieving complete mixtures, and in obtaining sufficient saraple quantities for analysis. The small beam diameter and relative volumes of active and inactive regions are both believed to be detrimental. The abortive attempts at separation indicate a need for considerably greater sophistication in the apparatus and care in aralytical measurements.

A new experiment has been designed to alleviate these difficulties, and to test the madel presented above. The new experiment, shown schematically in Fig. 6, will use a $3.65 \mathrm{~m}$ cell with the inactive volume reduced to almost zero. Small samples will be withdrawn from each end 


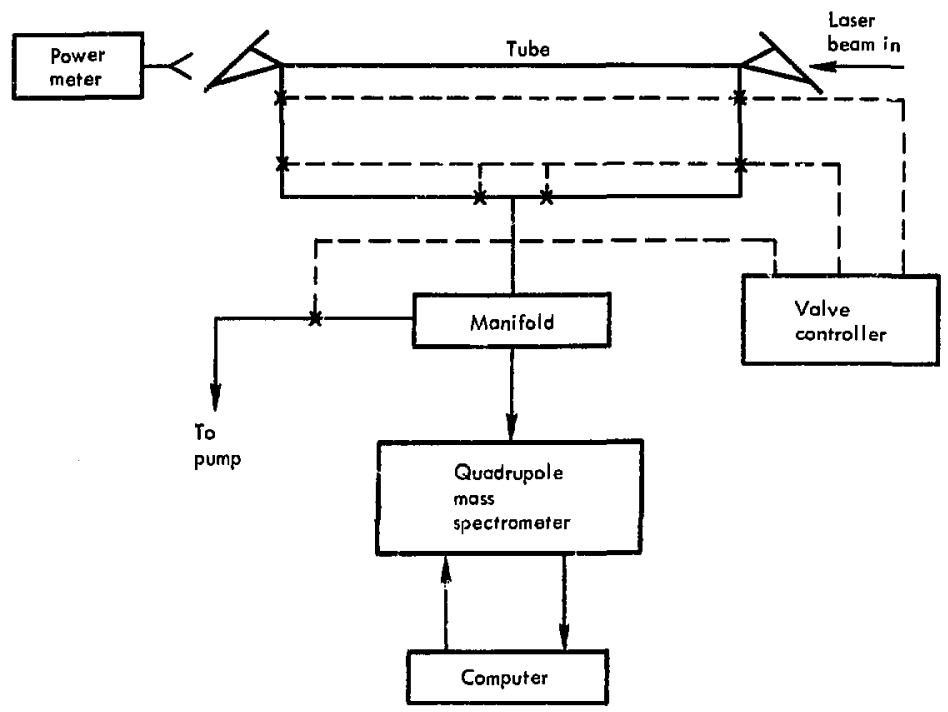

Fig. 6. Experimental apparatus for laser separation of binary gas samples.

simultaneougiy and analyzed on- line in a computer-controlled mass spectrometer. A new laser with $\approx 2 \mathrm{~cm}$ beam diam has bcen constructed so the test cell will be filled by the beam. Approximately $100 \mathrm{~W}$ cw is expected to be available. Premixed gases will be used. It has been shown ${ }^{8}$ that the $Q(12,2)$ line of the $v_{3}(1-0)$ band of ${ }^{12} \mathrm{CH}_{3} \mathrm{~F}$ lies within $44 \mathrm{MHz}$ of the $\mathrm{P}(20)$ line of the $9.4 \mu$ band of the $\mathrm{CO}_{2}$ laser. This transition has been chosen for initial tests. However, it has been shown ${ }^{9}$ that the relaxation from the upper state of this transition is rather slow; in fact, higher levels are rapidly populated where fluorescence occurs. We propose to use $\mathrm{N}_{2} \mathrm{O}$ as a buffer gas and use the 100 state of $\mathrm{N}_{2} \mathrm{O}$ as a resonance relaxation pathway from the $2 \nu_{3}$ state of ${ }^{12} \mathrm{CH}_{3} \mathrm{~F}$. In this case $\tau_{\text {eff }}\left(\mathrm{CH}_{3} \mathrm{~F}\right)$ should be greatly enhanced by the very large number of relaxation pathways through the rotational sublevels of $\mathrm{N}_{2} \mathrm{O}$. Since laser action is known to occur from this vibrational level of $\mathrm{N}_{2} \mathrm{O}$, some enhancement may be derived from the subsequent stimulated emission in $\mathrm{N}_{2} \mathrm{O}$. These gases are well behaved in regard to wall adsorption and clarity of mass spectra.

In order to test the theoretical model which we have presented, the following procedure will be followed. Samples of less than $1 \mathrm{~cm}^{3}$ volume will be drawn off sirultaneously from both ends before irradiation. After a suitable equilibrium time, when these samples show constant 
mass ratios of $\mathrm{CH}_{3} \mathrm{~F}$ and $\mathrm{N}_{2} \mathrm{O}$ at both ends, irradiation will commence. Samples will be drawn off at short intervals at first, and longer intervals later, in order to test the time dependence shown in Fig. 3. The val're and sampling controller in Fig. 6 will insure that pumpout and sampling procedures are repeated exactly for each sample. Each sample will then be analyzed several times in the mass spectrometer. In tests of the analytical procedure, expected variations in measured ratios have been found to remain less than $0.1 \%$.

\section{PULSED EXPERIMENTS}

Several recent studies $7,10,11$ of transient effects in the inirared and microwave spectral regions have produced results of interest. In particular, the expression for the population difference, $\Delta N$, between two levels for transient $a b-$ sorption is given by Eq. (30) of Ref, 10 as:

$\Delta N(t)=\Delta N_{0} / \Omega^{2}\left[(\Delta \omega)^{2}+k^{2} E^{2} \cos s t\right]$

where $\Delta \omega=\left(\omega-\omega_{0}\right)$ is the difference between the exiting and resonant absorption frequencies,

$\Omega=\left[\mathrm{k}^{2} \mathrm{E}^{2}+(\Delta \omega)^{2}\right]^{1 / 2}, \mathrm{k}=2|\langle\mathrm{a}|\mu| \mathrm{b}\rangle| \mathrm{h}$

and $E$ is the electric field intensity of the exciting radiation. When the pulse length (T) is such that

$$
\int_{0}^{\mathrm{T}} \Omega d t=\pi
$$

then $\Delta N(T)=-\Delta N$ and the populations are exactly inverted. This suggests that the use of the " $\pi$ " pulse allows the opportunity of involving essentially all the molecules in the lower state in the momen- tum transfer process. If, in addition, the " $\pi$ " pulse is immediately reflected back up the tube, stimulated emissions would revert the populations back to equilibrium and result in the transfer of two quanta of momentum to eash molecule. This would also make possible the recovery and reuse of the energy contained in the " $\pi$ " pulse.

Under these conditions, one may arrive at a molecular flux in analogy with the $\mathrm{N}_{\mathrm{o}} \cdot \mathrm{V}_{\mathrm{x}}$ used above. In this case the flux may be arrived at as the product of the number of molecules absorbing radiation per second and the average distance each moves per absorption. The number of molecules involved is $\Delta N_{o} \cdot r$, where $r$ is the pulse rate. The average distance each moves per pulse is

$$
v_{x} t_{k} \text { or } 2 h \tau_{k} / M \lambda \text {. }
$$

Then:

$$
\phi_{\pi}=\frac{2 \Delta \mathbf{n}_{o}{ }^{r h} T_{k}}{\lambda M} .
$$

In order to compare this with the $\mathrm{cw}$ experiment we may arrive at a $\phi_{\mathrm{cw}}$ in a sinnilar manner.

The number of absorptions per second is merely the power absorbed divided by he $/ \lambda$ or

$$
N_{o}=W_{c w}[1-\exp (-\gamma \ell)] \frac{\lambda}{h c}
$$

for the unsaturated case, so

$$
\phi_{\mathrm{cW}}=W_{\mathrm{cw}} \frac{\mid 1-\exp (-\gamma \ell)] \tau_{\mathrm{k}}}{M c}
$$

and we then bave

$$
\frac{\phi_{\pi}}{\phi_{\mathrm{cw}}}=\frac{2 \Delta \mathrm{N}_{\mathrm{o}} \mathrm{rhc}}{\left.\mathrm{W}_{\mathrm{o}}[1-\exp (-\gamma])\right] \lambda} .
$$

Recognizing that the average power, $W_{\pi}$, absorbed in the " $7 "$ "pulse experiment is 


$$
W_{\pi}=\Delta N_{0}+h c / \lambda
$$

we have

$$
\frac{\phi_{\pi}}{\phi_{\mathrm{cw}}}=\frac{2 \mathrm{w}_{\pi}}{\left.\mathrm{w}_{\mathrm{cw}}[1-\exp (-\gamma)]\right]} .
$$

Assuming that the same average power is available for both experiments, and the length and conditions of saturation have been optimized for the $\mathrm{cw}$ experimant so that essentially all of the cw power is absorbed, we have $\phi_{\pi} / \phi_{\mathrm{cw}}=2$. However, one important difference exists. In particular, it is possible to reccver the energy present in the " $\pi$ " pulse since, after reflection, the pulse will grow due to the inverted absorber population. This energy then becomes reusable some number of times (N) with the laser input making up the losses. The advantage then is $2 \mathrm{~N}$.

\section{INTERRUPTED CW METHOD}

The proposed model suggests, as shown in Figs. 2 and 3 , that relative concentration will change rapidly near the ends of the absorption cell. This indicates that a sample consisting of a short length of the modified mixture may be drawn off at short time intervals. Since the mixture has not been modified at the center, the total quantity of mixture may be replenished at that point without disturbing the system. We propose that efficiency would be greatly enhanced by withdrawing the partially separated mixture at short intervals, in contrast to a static system where little rurther improvement in concentration ratios occurs at the ends with extended exposure, From Fig. 2 we see that for a I-m tube the ratio of concentration at the far end to that at the near end is piedicted to be 4.25 after $1000 \mathrm{sec}$. However, the same figure shows a ratio of 2.33 after only $200 \mathrm{sec}$, a factor of less than $2 \mathrm{im}-$ provement with a factor of 5 increase in time. Cascading two cells would result in a ratio of 4.67 in the shorter time. Since efficiency deponds directly upon the separation rate, it would be greatly enhanced by removing the separated molecules near the end at very short intervals and relying upon exter'ral pumping to move the mixture into and out of the cascaded colls.

\section{Applications}

The application of radiation-pressureinduced separations receiving the greatest recent $r^{+t i}$,ention is isotope separation, However, other applications are also of great importance, particularly that of isomer separation. One may also speculate concerning the possibllity of removal of the products of a reversible chemical reaction, thus driving the reaction in the desired direction. One may also influence the concentration of particular molecular states near one end of the tube, again nodifying reaction rates, or producing particular states for other studies.

Isotopic mixtures would be an obvious application of bulk molecular separation by radiation pressure. In a 1-m tube, an enrichment factor of over 10 would be achieved in less than $30 \mathrm{~min}$ with the $\mathrm{cw}$ laser and twice that for a pulsed laser. Furthermore, this could be accomplished using several Torr of material; i,e., 
m moles per hour could be easily separated. We expect that, at least for the lighter elements, the whole procedure would be physically simpler and less costly than conventional separation schemes.

Another important application would be the scparation of optical isomers, Current technology involves either complex stereo-specific synthesis, starting from natural products, or synthesis of diastereomers followed by laborious physical separation. Many organic com- pounds of potential interest in Finnthetic and biochemical applications have sufficient vapor pressure and a different dichroic absorption to be amenable to a bulk separation by radiation pressure. For this application, the shorter lifetime and greater momentum transfer associated with visible and $u v$ radiation would be advantageous, while the elimination of strict monochromaticity and tunability would make such a process feasible even with conventional light sources.

\section{Summary}

We have considered the feastbility of ubtaining physical separations of large quantities of gas by the application of resonant radiation pressure through a bulk sample. Preliminary experimental attempts at producing and detecting such separations, while not conclusive, have stimulated further investigation and have provided information for more optimal experimental conditions.

The model developed in this paper shows that jnolecular motion in a closed tube under the influence of an applied force is similar in many respects to electron "mobility" in a conductor. Thermodynamic calculations show that the process should be efficient and substantlal.

Refined experiments have been suggested which should prove the feasibility for bulk separations of an arsorbing molecular species from a nonabsorbing bufter. These experiments could then be extended directly to separating isotopes and isomers. Some interesting adaptations of this technique have been considered which could lead to large, efficient separations.

\section{Acknowledgments}

We are gratcful to Jack W. Frazer for suggestions reiating in the pulaed experi- ments and to Klaus Ernst for discussions about the analytical considerations. 


\section{References}

1. A. Ashkin, Phys. Rey, Lett. 24, 156-159 (1970).

2. J. L. Picque and J. L. Vialle, Opt. Commun. 5, 402-406 (1972).

3. R. Schieder, H. Walther and L. Woste, Opt. Commun. S, 337-340 (1972).

4. A. F. Bernhardt, D.E. Duerre, J.R. Simpson and L. L. Wood, Appl. Phys. Lett. 25, 617-620 (1974).

5. J. Gelbwachs and T.S. Hartwick, IEEE Quantum Electron., Jan. 1975, 52-54.

6. R. B. Bird, W. E. Stewart and E. N. Lightfoot, Transport Phenomena, (John Wiley \& Sons, N.Y., 1960), Chapters 16-18.

7. J, C, McGurk, T. G. Schmalz, and W, H. Flygare, Adv. Chem. Phys., Vol, 25.

8. S. M. Freund, G. Duxbury, M. Romheld, J. T. Tiedje and T. Oka, J. Mol. Spectrosc. 52, 38 (1974).

9. E. Weitz and G.W. Flynn, J. Chem. Phys. 58, 2781 (1973).

10. J.C. McGurk, R, T. Hofmann and W.H, Flygare, J, Chern. Phys. 60, 2922 (1974).

11. R. G. Brewer and R. L. Shomaker, Phys. Rev, Lett. 27, 631 (1971). 